\title{
Variation of corneal refractive index with hydration
}

\author{
Young L Kim, Joseph T Walsh Jr, Thomas K Goldstick \\ and Matthew R Glucksberg \\ Biomedical Engineering Department, Northwestern University, Evanston, IL 60208, USA \\ E-mail: younglae@northwestern.edu
}

Received 15 November 2003, in final form 14 January 2004

Published 13 February 2004

Online at stacks.iop.org/PMB/49/859 (DOI: 10.1088/0031-9155/49/5/015)

\begin{abstract}
We report the effect of changes in the corneal hydration on the refractive index of the cornea. Using optical coherence tomography (OCT), the geometrical thickness and the group refractive index of the bovine cornea were derived simultaneously as the corneal hydration was varied. The corneal hydration was then calculated from the corneal thickness. The group refractive index of the cornea increased non-linearly as the cornea dehydrated. In addition, a simple mathematical model was developed, based on the assumption that changes in corneal hydration occur only in the interfibrilar space with constant water content within the collagen fibrils. Good agreement between the experimental results and the mathematical model supports the assumption. The results also demonstrate that the measurement of refractive index is a quantitative indicator of corneal hydration.
\end{abstract}

\section{Introduction}

The cornea consists of three basic layers: the epithelium, the stroma and the endothelium. The stroma constitutes $\sim 90 \%$ of the thickness of the cornea. The stromal lamellae, which are long, parallel, cylindrical collagen fibrils, are embedded in a ground substance whose refractive index is lower than that of the collagen fibrils. The normal cornea contains $76.2 \%$ water by weight (Leonard and Meek 1997). A constant corneal hydration is essential for the maintenance of its transparency. A swollen corneal stroma contains irregular regions in which refractive index fluctuations scatter light and cause the cornea to opacify (Benedek 1971).

Changes in the corneal thickness are known to be proportional to changes in corneal hydration (Hedbys and Mishima 1966). Accurate measurement of the corneal hydration and thickness is useful for both diagnostic and monitoring purposes as well as surgical planning. For example, cornea thickness decreases with prolonged contact lens wear because of hypoxia (Liu and Pflugfelder 2000). In excimer laser keratorefractive surgery, $53 \mu \mathrm{m}$ of central stromal tissue in a $4 \mathrm{~mm}$ diameter area is removed to correct -10 diopters of myopia (Munnerlyn et al 1988); accurate measurement of corneal thickness may improve 
outcome in these surgical procedures. In addition, corneal hydration itself affects the excimer laser ablation rate (Dougherty et al 1994). The corneal thickness affects the measurement of intraocular pressure (IOP), which is a primary factor in the diagnosis and the treatment of glaucoma. With the Goldmann applanation tonometer, a decreased corneal thickness after eximer myopic photorefractive keratectomy (PRK) leads to underestimation of IOP by $\sim 2.40 \mathrm{mmHg}$ (Abbasoglu et al 1998), where normal IOP is $\sim 15 \mathrm{mmHg}$. Similarly, laser in situ keratomileusis (LASIK) causes a decrease of $1.0 \mathrm{mmHg}$ per $37.8 \mu \mathrm{m}$ of removed cornea (Emara et al 1998).

A new optical imaging technique, optical coherence tomography (OCT), has been developed and applied to measure thickness in biological samples, especially in the eyes with micron-resolution. The resolution is defined as the coherence length of the light source. The coherence length, $l_{c}$, is inversely proportional to the width of the emission spectrum of the light source (Fercher 1996, Huang et al 1991a). In order to achieve high resolution, OCT uses a partially coherence (or low-coherence) light source, which means that the light source emits light in a broad range of wavelengths. When a sample consists of a dispersive medium (dispersion is a characteristic of a material that presents the wavelength dependence of the velocity of light propagation), each emitted wavelength component has a different velocity in a material. A group of different wavelengths (i.e. a wave packet) propagates as a group velocity, $v_{g}=c / n_{g}$ ( $c$ is the velocity of light in vacuum and $n_{g}$ is the group refractive index of the medium) through the material (Hecht 1987). The relationship between the group refractive index, $n_{g}$, and the phase index, $n_{p}$, is $n_{g}=n_{p}-\lambda \frac{\mathrm{d} n_{p}(\lambda)}{\mathrm{d} \lambda}$, where $\frac{\mathrm{d} n_{p}(\lambda)}{\mathrm{d} \lambda}$ is chromatic dispersion (Neumann 1988). The partially coherence light forms a wave packet that sees the group refractive index of the sample rather than the phase refractive index. Therefore, a measured thickness in OCT is the product of the geometrical thickness and the group refractive index of the sample. The group refractive index must be used rather than the phase refractive index when the geometrical thickness needs to be obtained. Since it was shown that the cornea is about five times more dispersive than water in the spectral range of $814 \mathrm{~nm}-$ $855 \mathrm{~nm}$ (Drexler et al 1998), $n_{p}$ is different from $n_{g}$ such that $n_{p}=1.3708$ and $n_{g}=1.3854$ at $814 \mathrm{~nm}$ (Hitzenberger 1991). Therefore, in this experiment, the group refractive index was measured instead of the phase refractive index.

OCT is useful for corneal thickness measurements with an accuracy of a few micrometres. The OCT-measured thickness is not, however, the geometrical thickness but rather the optical thickness: the geometrical thickness is calculated by dividing the optical thickness by the refractive index. Previous measurements of corneal thickness using OCT-based techniques assumed that the refractive index was constant (Bechmann et al 2000, 2001, Feng et al 2001, Hitzenberger et al 1994, Huang et al 1991b, Radhakrishnan et al 2001). Hydration or dispersion may alter the corneal refractive index. Although OCT can yield measurements of optical corneal thickness with micron-resolution, to achieve similar accuracy for the geometric thickness, the group refractive index of the cornea must be measured. Here we report the effect of changes in thickness and hydration on the group refractive index for excised bovine cornea.

\section{Materials and methods}

\subsection{System description}

The light source was a superluminescent diode (SLD) (Superlum Ltd., Moscow, Russia) with central wavelength $\lambda_{s}$ of $819.9 \mathrm{~nm}$, full width spectral bandwidth $\Delta \lambda$ of $21.9 \mathrm{~nm}$ at half maximum, spectral ripple of $0.4 \%$ and maximum output power of $1.80 \mathrm{~mW}$ at $140 \mathrm{~mA}$ SLD 
current. The measured coherence length of the light source was $18 \mu \mathrm{m}$, which is close to theoretical coherence length of $16.5 \mu \mathrm{m}\left(l_{c}=\frac{2 \ln 2}{\pi} \cdot \frac{\lambda_{s}^{2}}{\Delta \lambda}\right)$ (Huang et al 1991a). The current and the temperature of the SLD were controlled by a PILOT-2 Driving Set (Superlum Ltd., Moscow, Russia). The light passed through an optical fibre to a 50/50 coupler (Fibre Optic Network Technology Co., Surrey, British Columbia, Canada) where it was split into the reference arm and the sample arm. The light in each arm was collimated with an aspheric lens (Thorlabs, Inc., Newton, NJ) placed immediately at the optical fibre end. The light in the reference arm was reflected by a corner cube retroreflector (Edmund Scientific, Co., Barrington, NJ) that was mounted on a voice coil scanning system (QuickScan, Physik Instrumente GmbH \& Co., Waldbronn, Germany). The scanning speed of the voice coil scanning system was set as $20 \mathrm{~mm} \mathrm{~s}^{-1}$, corresponding to $49 \mathrm{KHz}$ modulation frequency. In the sample arm, the light was reflected by the internal structure of the sample. When a cross section of the cornea was imaged, transverse scans were performed using an XY scanner (GSI lumonics, Watertown, MA). The light reflected from the sample and reference arms was recombined at a photodetector (New Focus, Inc., Santa Clara, CA). The $-3 \mathrm{~dB}$ cut-off frequencies of the detector passband were at $30 \mathrm{KHz}$ and $100 \mathrm{KHz}$. The output signal was sent to a lock-in amplifier (SRS Stanford Research Systems, Sunnyvale, CA) to obtain the envelope of the interference signal. The time constant, the sensitivity and the low-pass-filter slope of the lock-in amplifier were set as $1 \times$ $10 \mu \mathrm{s}, 1 \times 1 \mathrm{~V}$ and $-24 \mathrm{~dB} /$ oct, respectively. A data acquisition board (National Instruments, Austin, TX) sampled the signal at $f_{s}=200 \mathrm{KHz}$.

\subsection{Simultaneous measurement of group refractive index and thickness}

The group refractive index and the thickness of samples were measured simultaneously by obtaining the optical thickness and the optical shift of the samples (Sorin and Gray 1992, Tearney et al 1995). First, a stationary mirror was placed in the sample arm (figure 1(a)). Given that the group refractive index of air is 1 , the geometric position of the mirror $\left(\mathrm{x}_{1}\right)$ was found. The sample, whose group refractive index, $n_{g}$, and geometrical thickness, $T$, were unknown, was then placed before the mirror in the sample arm (figure 1(b)). One notes that the peaks from the anterior $\left(\mathrm{x}_{2}\right)$ and the posterior $\left(\mathrm{x}_{3}\right)$ surface of the sample appeared with optical thickness, $n_{g} T$, and that the position of the mirror $\left(\mathrm{x}_{4}\right)$ was shifted to the right by $n_{g} T-T$ due to the sample whose group refractive index is greater than that of air. The optical thickness measurement and the optical shift measurement are required to calculate the thickness and the group refractive index of the sample. The calculation of the geometrical thickness and the group refractive index proceeds as follows. The optical thickness, OT, of the sample is

$$
\mathrm{OT}=n_{g} T
$$

where $n_{g}$ is the group refractive index and $T$ is the sample thickness. The apparent position of the mirror is moved to the right by the sample. The optical shift, OS, of the stationary mirror is

$$
\mathrm{OS}=n_{g} T-T
$$

Now we have two equations with two knowns, OT and OS, and two unknowns, $n_{g}$ and $T$. If we divide equation (2) by equation (1), $n_{g}$ is solved by

$$
n_{g}=\frac{\mathrm{OT}}{\mathrm{OT}-\mathrm{OS}} .
$$




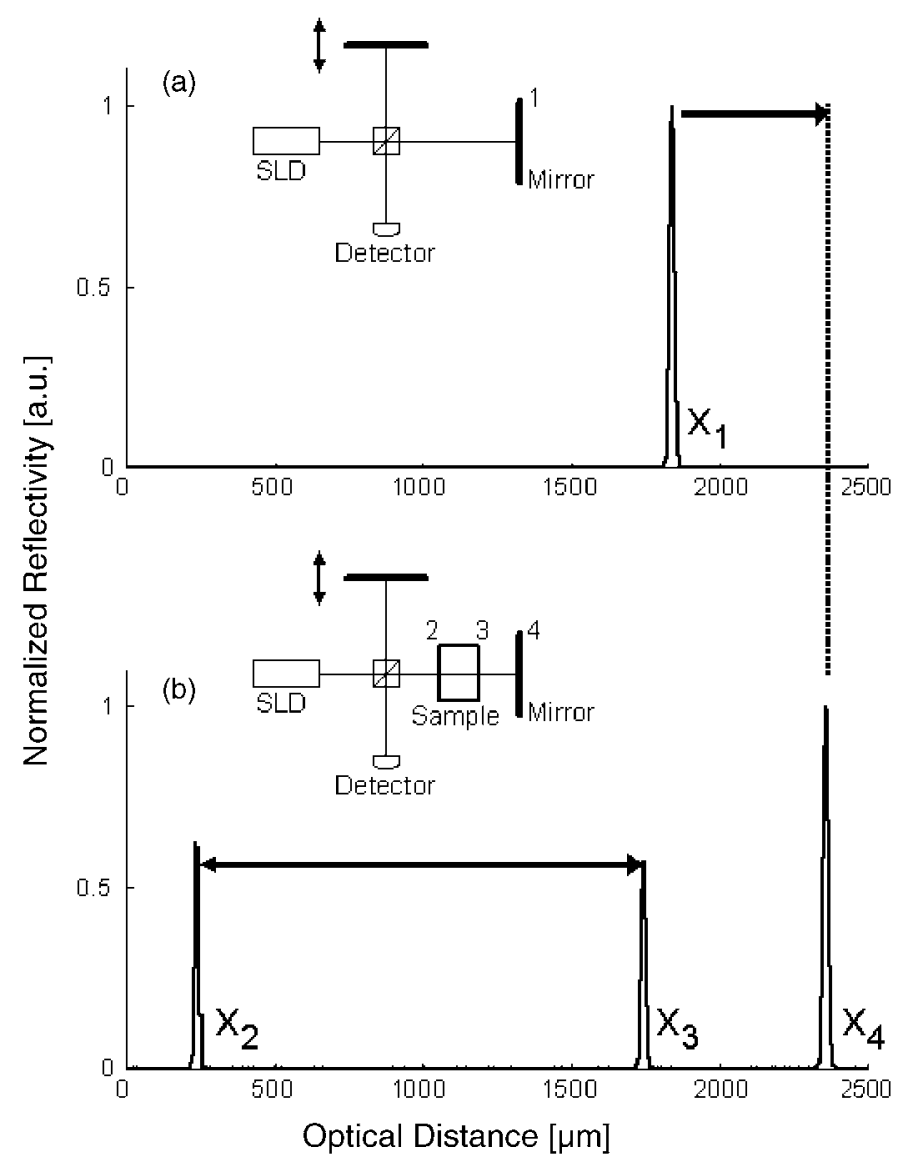

Figure 1. (a) An A-scan measures the location $\left(x_{1}\right)$ of the fixed mirror without a sample in the sample arm. (b) A sample, whose thickness is $T$ and the group refractive index is $n_{g}$, is placed in front of the fixed mirror. The first two peaks $\left(\mathrm{x}_{2}\right.$ and $\left.\mathrm{x}_{3}\right)$ are the interference signals from the anterior and posterior surface of the sample. The distance from $\mathrm{x}_{2}$ to $\mathrm{x}_{3}$ is the optical thickness of the sample, OT $=n_{g} T$. The apparent location of the mirror $\left(\mathrm{x}_{4}\right)$ is shifted from $\mathrm{x}_{1}$ by the sample, $\mathrm{OS}=n_{g} T-T$.

Then, the geometrical thickness, $T$, is easily calculated as

$$
T=\frac{\mathrm{OT}}{n_{g}}=\mathrm{OT}-\mathrm{OS} .
$$

Accuracy and repeatability were tested with a microscope slide (Fisher Scientific, Pittsburgh, PA). First, the geometrical position of the fixed mirror in the sample arm was found without the microscope slide. 50 A-scans were collected and saved in the computer. Second, the slide was placed before the mirror in the sample arm, and $50 \mathrm{~A}$-scans were collected and saved. From equations (3) and (4), the group refractive index and the geometrical thickness were calculated such that $T=1024(\mathrm{SD} \pm 1) \mu \mathrm{m}$ and $n_{g}=1.516(\mathrm{SD} \pm 0.001)$. In addition, the geometrical thickness of the slide was measured using a micrometer. The thickness measured with the micrometer was 1025 (SD \pm 5$) \mu \mathrm{m}$ with the sample size of 10. A $t$-test was used to test whether the two measured thicknesses were equal statistically. The calculated $t$-value was 0.63 and the corresponding $P$-value (two-tailed) was 0.53 , 


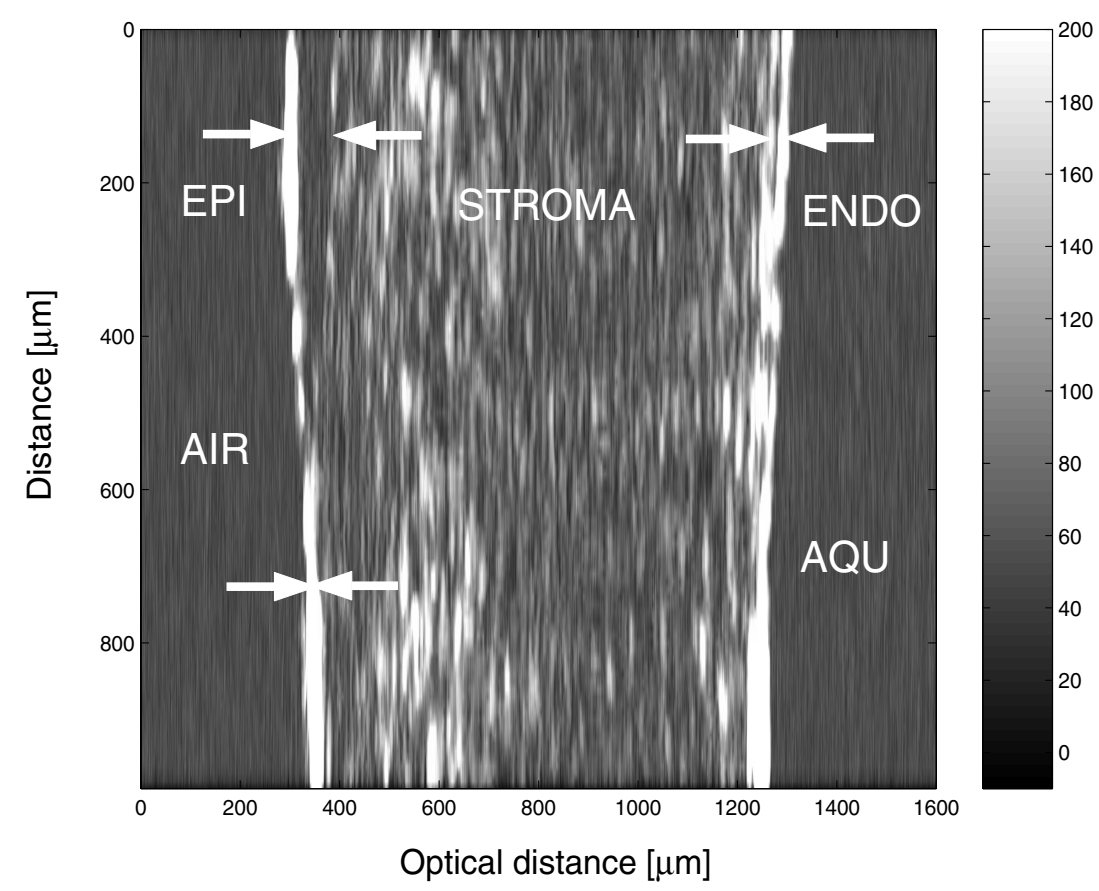

Figure 2. OCT image of the bovine cornea. EPI, STROMA and ENDO denote the epithelium, the stroma and the endothelium, respectively. The left side of the cornea is air (AIR) and the right side of the cornea is the aqueous humour (AQU). The epithelium is removed in the lower part of the cornea (see the arrows in the lower left corner). The strongest reflections are from the differences in refractive indices at the air-tissue and tissue-aqueous interfaces.

for 58 degrees of freedom. Therefore, the null hypothesis that the two measurements are equal was accepted.

\subsection{In vitro measurement}

Twenty calf eyes were collected from a local slaughterhouse and brought to our laboratory within $1 \mathrm{~h}$ postmortem. The epithelium of each eye was removed by scraping with a surgical blade (Feather Safety Razor Co., Ltd., Osaka, Japan). Figure 2 shows an OCT image of the bovine cornea. The epithelium of the lower part of the cornea was removed while that of the upper part was intact. Figure 3 shows two corresponding A-scans from figure 2 at the $100 \mu \mathrm{m}$ and $700 \mu \mathrm{m}$ vertical positions. The distance between the second peak and the last peak of the upper A-scan is almost equal to the distance between the first peak and the last peak of the lower A-scan. The optical thickness of the epithelium was $87 \mu \mathrm{m}$, which is consistent with the assumption that only the epithelium was removed by scraping.

Five eyes were used to find the geometrical thickness and the group refractive index under normal hydration conditions. The following steps were repeated for each of the five eyes. First, the epithelium was removed by scraping with the surgical blade, and a disc about $10 \mathrm{~mm}$ in diameter was cut from the central region of the cornea. Then, ten A-scans of the position of the fixed mirror in the sample arm were collected. Finally, the cornea, mounted in a holder designed to keep the cornea in its naturally circular shape, was placed before the fixed mirror and ten A-scans of three different positions in the central region were collected.

Fifteen eyes were used to determine the effect of hydration on the group refractive index and the corneal thickness. The following steps were repeated for each of the 15 eyes. First, the 


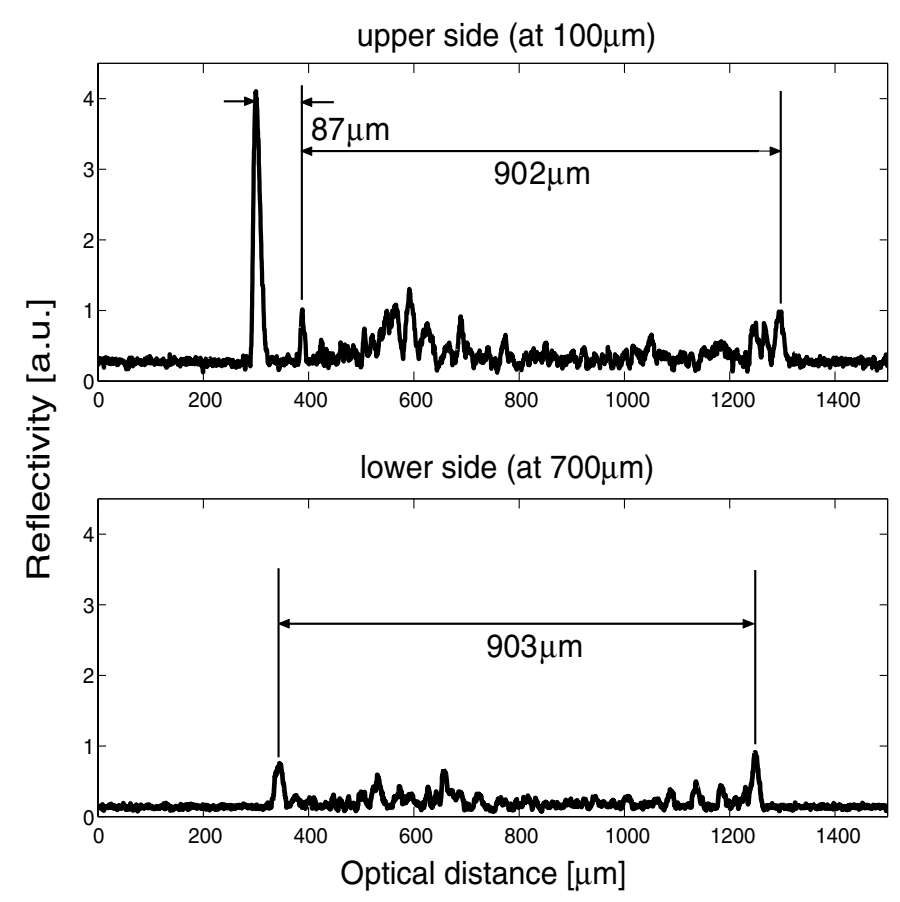

Figure 3. Upper: An A-scan at $100 \mu \mathrm{m}$ transverse position (horizontal cross-section) from figure 2. Lower: An A-scan at $700 \mu \mathrm{m}$ transverse position from figure 2. Note the nearly identical stromal thickness.

epithelium was removed with the method described above. To increase the corneal hydration to various levels, each eye was placed in a beaker containing $50 \mathrm{ml}$ of physiological saline solution ( $0.9 \% \mathrm{NaCl}$ solution) for various periods (from $3 \mathrm{~h}$ up to $5 \mathrm{~h}$ ). Next, a $10 \mathrm{~mm}$ diameter disc was cut from the central corneal region. To change the corneal hydration uniformly across the corneal thickness, reducing the dehydration rate, three different humidity chambers were prepared. An excess of a water soluble salt in contact with its saturated solution in a closed chamber produces a constant relative humidity (Greenspan 1977). Each chamber contained saturated solutions of one of the compounds: $\mathrm{K}_{2} \mathrm{SO}_{4}, \mathrm{KCl}$ and $\mathrm{NaCl}$ for relative humidities of $97 \%, 84 \%$ and $75 \%$ at $25{ }^{\circ} \mathrm{C}$, respectively (Wexler 2000). After the corneal disc was cut, it was placed in a small petri dish and then placed in the closed $\mathrm{K}_{2} \mathrm{SO}_{4}$ chamber to be dehydrated uniformly. After $18 \mathrm{~h}$ in the $\mathrm{K}_{2} \mathrm{SO}_{4}$ chamber, the cornea was placed in the holder before the fixed mirror and ten A-scans of three different positions in the central region of the cornea were collected. After the data collection, the same cornea was placed in the $\mathrm{KCl}$ chamber for $10 \mathrm{~h}$, and then measured data were collected. Next, after $10 \mathrm{~h}$ in the $\mathrm{NaCl}$ chamber, the data were collected. Finally, ten A-scans without the cornea were collected to determine the position of the mirror. The laboratory room temperature was $25^{\circ} \mathrm{C}$ and the relative humidity in the laboratory was $54 \%$.

The corneal hydration, which is defined by the ratio of the water weight to the completely dry weight of the cornea, was calculated from the known linear relationship between bovine corneal hydration and thickness (Fatt and Goldstick 1965, Hedbys and Mishima 1962, 1966),

$$
H=5.3 \times 10^{-3} \cdot T-0.67
$$

where $H$ is the hydration of the corneal stroma and $T$ is the thickness of the corneal stroma in $\mu \mathrm{m}$. 


\section{Mathematical model}

A mathematical model of the relationship between the corneal refractive index and hydration was developed. The refractive index of the cornea, $n_{\mathrm{co}}$, is given by Maurice (1957)

$$
n_{\mathrm{co}}=n_{\mathrm{cf}} \cdot f_{\mathrm{cf}}+n_{\mathrm{gs}} \cdot f_{\mathrm{gs}}
$$

where $n_{\mathrm{cf}}$ is the refractive index of the collagen fibrils, $n_{\mathrm{gs}}$ is that of the ground substance, $f_{\mathrm{cf}}$ is the volume fraction of the collagen fibrils and $f_{\mathrm{gs}}$ is that of the ground substance $\left(f_{\mathrm{cf}}+f_{\mathrm{gs}}=1\right)$. It is assumed that the drying of the cornea dehydrates only the ground substance so that the corneal refractive index increases without changing water content of the collagen fibrils, in which water is tightly bound. When the ground substance is completely dehydrated, the corneal refractive index will be close to that of the collagen fibrils, because the volume fraction of the collagen fibrils and that of the dehydrated ground substance are found to be $\sim 30 \%$ and $\sim 10 \%$, respectively (Leonard and Meek 1997). Similarly, the swelling of the cornea takes place only in the ground substance, keeping the hydration of the collagen fibrils constant. The volume fractions of the collagen fibrils and the ground substance are

$$
f_{\mathrm{cf}}=\frac{V_{\mathrm{cf}}}{V_{\mathrm{cf}}+V_{\mathrm{gs}}} \quad \text { and } \quad f_{\mathrm{gs}}=\frac{V_{\mathrm{gs}}}{V_{\mathrm{cf}}+V_{\mathrm{gs}}}
$$

where $V$ denotes the volume of each component. The volume fraction, $V_{\mathrm{cf}}$, and the refractive index, $n_{\mathrm{cf}}$, of the collagen fibrils are constant over changes in the corneal hydration because the hydration takes place only in the ground substance. The ground substance is considered as a mixture of dry material and water. The volume of the ground substance, $V_{\mathrm{gs}}$, is expressed by

$$
V_{\mathrm{gs}}=V_{0 \mathrm{gs}}+V_{\mathrm{H}_{2} \mathrm{O}}
$$

where $V_{0 \mathrm{gs}}$ is the volume of the ground substance when the ground substance is completely dehydrated and $V_{\mathrm{H}_{2} \mathrm{O}}$ is the water content of the ground substance. The refractive index of the ground substance, $n_{\mathrm{gs}}$, can be decomposed such that

$$
n_{\mathrm{gs}}=n_{0 \mathrm{gs}} \frac{V_{0 \mathrm{gs}}}{V_{\mathrm{gs}}}+n_{\mathrm{H}_{2} \mathrm{O}} \frac{V_{\mathrm{H}_{2} \mathrm{O}}}{V_{\mathrm{gs}}}
$$

where $n_{0 \mathrm{gs}}$ is the refractive index of the dry ground substance and $n_{\mathrm{H}_{2} \mathrm{O}}$ is that of water. Then, $V_{\mathrm{H}_{2} \mathrm{O}}$ is given by the definition of the hydration:

$$
V_{\mathrm{H}_{2} \mathrm{O}}=H \cdot\left(V_{0 \mathrm{gs}}+V_{\mathrm{cf}}\right)
$$

where $H$ is the corneal hydration. Substituting equations (7)-(10) into (6), equation (6) is rewritten as a function of $H$ :

$n_{\mathrm{co}}(H)=n_{\mathrm{H}_{2} \mathrm{O}}+\frac{V_{\mathrm{cf}} \cdot\left(n_{\mathrm{cf}}-n_{\mathrm{H}_{2} \mathrm{O}}\right)+V_{0 \mathrm{gs}} \cdot\left(n_{0 \mathrm{gs}}-n_{\mathrm{H}_{2} \mathrm{O}}\right)}{(H+1) \cdot\left(V_{0 \mathrm{gs}}+V_{\mathrm{cf}}\right)}=a+\frac{b}{H+1}$

where $a$ and $b$ are constants. Using equation (5), the corneal refractive index can be expressed as a function of $T$ :

$$
n_{\mathrm{co}}(T)=a+\frac{b}{5.3 \times 10^{-3} \cdot T+0.33} .
$$




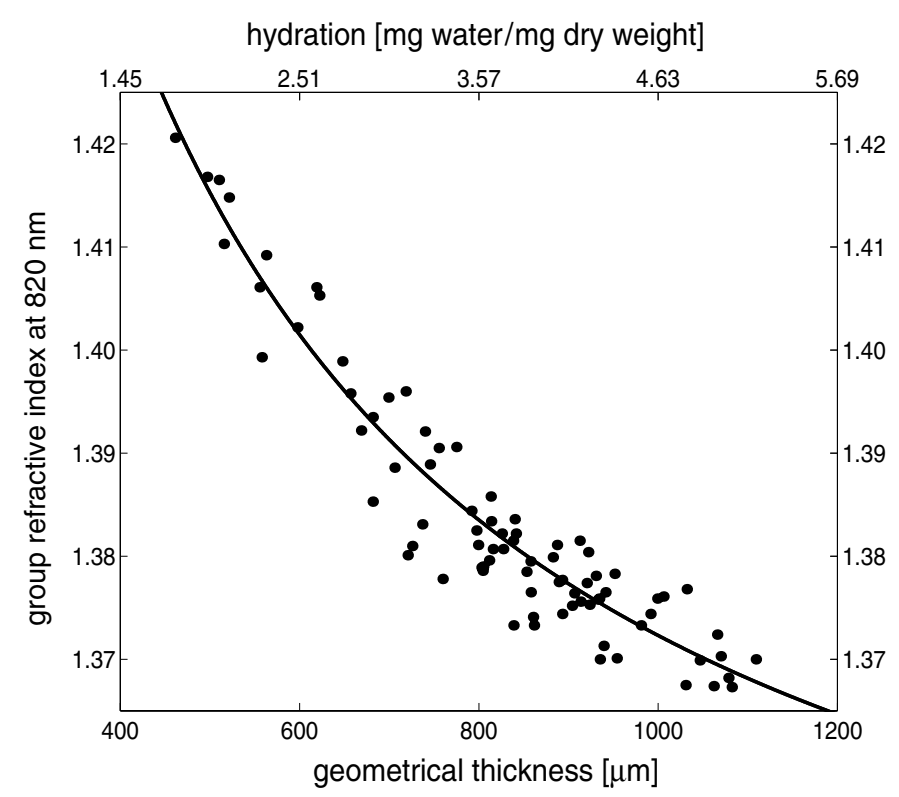

Figure 4. The group refractive index of the cornea versus the geometrical thickness and the hydration. Group refractive index and thickness were calculated using equations (3) and (4), respectively. The hydration was calculated from the geometrical thickness using equation (5). The data were fit with $n_{g}=a+b /(H+1) . \quad a=1.324 \pm 0.002$ (mean $\pm \mathrm{SE}, n=82$ ) and $b=0.272 \pm 0.009$ (mean $\pm \mathrm{SE}, n=82$ ) were calculated by least squares regression.

\section{Results}

The group refractive index and the thickness of the five corneas under normal condition were $1.380(\mathrm{SD} \pm 0.001)$ and $782(\mathrm{SD} \pm 57) \mu \mathrm{m}$, respectively. The group refractive index of all 20 eyes at various thicknesses is shown in figure 4 . Figure 4 also illustrates the relationship between the group refractive index and the hydration (the top horizontal axis), which was calculated from the thickness using equation (5). The group refractive index increases as the cornea is dehydrated. The solid line is a fit with the mathematical model, $n_{\mathrm{co}}(H)=a+b /(H+1)$, by the method of least squares: $a=1.324 \pm 0.002$ (mean \pm standard error, $n=82)$ and $b=0.272 \pm 0.009$ (mean \pm SE, $n=82)$.

\section{Discussion}

When one studies changes in the corneal thickness with an OCT system by converting the optical thickness to the geometrical thickness, one cannot assume that the refractive index is constant. For example, in corneal edema, the geometrical thickness that is calculated by dividing the optical thickness by a constant refractive index can be smaller than the actual geometrical thickness because the group refractive index is decreased. Feng et al (2001) measured overnight swelling and deswelling of the cornea with OCT, using a constant cornea refractive index and assuming that the constant refractive index would lead to only $1 \%$ error in the thickness measurement. Although the percentage of the error is small, a constant refractive index can induce more than a $10 \mu \mathrm{m}$ error. For instance, suppose that the optical thickness of the cornea is $700 \mu \mathrm{m}$, the geometrical thickness can be either $511 \mu \mathrm{m}$ by the refractive index of 1.37 or $500 \mu \mathrm{m}$ by the refractive index of 1.40 . Therefore, the variation of the group 
refractive index with hydration must be considered to take advantage of the accuracy of the OCT-measured thickness.

The parameter, $a=1.324 \pm 0.002$ (mean $\pm \mathrm{SE}, n=82$ ), in equation (12) corresponds to the group refractive index of water since low-coherence light forms a wave packet that sees the group refractive index of the cornea in OCT. The phase refractive index of water is 1.329 at $825 \mathrm{~nm}$ and $25^{\circ} \mathrm{C}$ (Hale and Querry 1973). Naturally, $a$ must be compared with the group refractive index of water at $820 \mathrm{~nm}, 25^{\circ} \mathrm{C}$, and a specific pressure. The refractive index of water is dependent on wavelength, temperature and pressure; further, the group refractive index of water may be different from the phase refractive index because water is dispersive.

One drawback of our method for measuring the refractive index and thickness is that it is invasive. Because of the two unknowns of the sample, $n_{g}$ and $T$, two equations and two measurements are required. The optical thickness of the sample is one measurement; the second is found from the shift of the position of the fixed mirror when the sample is placed immediately in front of the fixed mirror. Since the opposite side of biological tissues is often inaccessible, this measurement has limitations for most noninvasive in vivo applications. We are currently considering a number of minimally invasive techniques to quantify the shift induced by the sample and to find the refractive index. Complete development of a clinical system must await these improvements, which could be used to quantify corneal thickness in vivo in human eyes.

\section{Conclusion}

The effect of the corneal hydration on the corneal refractive index was studied by deriving the group refractive index and the geometrical thickness simultaneously. The group refractive index of the cornea increases non-linearly as the cornea dehydrates. Good agreement with the mathematical model supports the idea that changes in hydration occur in the ground substance without changing water content of the collagen fibrils. The results demonstrate that the refractive index can be a potential tool for monitoring the corneal hydration, which is a crucial factor clinically, and that the measurement of the corneal thickness can be improved by considering the accurate refractive index.

\section{Acknowledgments}

This work was supported by grants from the National Institutes of Health (NIH EY 13015 and NIH EY 09714).

\section{References}

Abbasoglu O E, Bowman R W, Cavanagh H D and McCulley J P 1998 Reliability of intraocular pressure measurements after myopic excimer photorefractive keratectomy Ophthalmology $1052193-6$

Bechmann M, Thiel M J, Neubauer A S, Ullrich S, Ludwig K, Kenyon K R and Ulbig M W 2001 Central corneal thickness measurement with a retinal optical coherence tomography device versus standard ultrasonic pachymetry Cornea $2050-4$

Bechmann M, Thiel M J, Roesen B, Ullrich S, Ulbig M W and Ludwig K 2000 Central corneal thickness determined with optical coherence tomography in various types of glaucoma Br. J. Ophthalmol. $841233-7$

Benedek G B 1971 Theory of transparency of the eye Appl. Opt. 10 459-73

Dougherty P J, Wellish K L and Maloney R K 1994 Excimer laser ablation rate and corneal hydration Am. J. Ophthalmol. 118 169-76

Drexler W, Hitzenberger C K, Baumgartner A, Findl O, Sattmann H and Fercher A F 1998 Investigation of dispersion effects in ocular media by multiple wavelength partial coherence interferometry Exp. Eye Res. 66 25-33 
Emara B, Probst L E, Tingey D P, Kennedy D W, Willms L J and Machat J 1998 Correlation of intraocular pressure and central corneal thickness in normal myopic eyes and after laser in situ keratomileusis J. Cataract Refract. Surg. 24 1320-5

Fatt I and Goldstick T K 1965 Dynamics of water transport in swelling membranes J. Colloid Sci. 20 962-89

Feng Y, Varikooty J and Simpson T L 2001 Diurnal variation of corneal and corneal epithelial thickness measured using optical coherence tomography Cornea 20 480-3

Fercher A F 1996 Optical coherence tomography J. Biomed. Opt. 1 157-73

Greenspan L 1977 Humidity fixed points of binary saturated aqueous solutions J. Res. Natl. Bur. Stand. A 8 89-95

Hale G M and Querry M R 1973 Optical constants of water in the 200-nm to 200- $\mu$ m wavelength region Appl. Opt. 12 555-63

Hecht E 1987 Optics (Reading, MA: Addison-Wesley) p 253

Hedbys B O and Mishima S 1962 Flow of water in the corneal stroma Exp. Eye Res. $1262-75$

Hedbys B O and Mishima S 1966 The thickness-hydration relationship of the cornea Exp. Eye Res. 5 221-8

Hitzenberger C K 1991 Optical measurement of the axial eye length by laser Doppler interferometry Invest. Ophthalmol. Vis. Sci. 32 616-24

Hitzenberger C K, Baumgartner A, Drexler W and Fercher A F 1994 Interferometric measurement of corneal thickness with micrometer precision Am. J. Ophthalmol. 118 468-76

Huang D, Swanson E A, Lin C P, Schuman J S, Chang W, Hee M R, Flotte T, Gregory K, Puliafito C A and Fujimoto J G 1991a Optical coherence tomography Science 254 1178-81

Huang D, Wang J, Lin C P, Puliafito C A and Fujimoto J G 1991b Micro-resolution ranging of corneal anterior chamber by optical reflectometry Lasers Surg. Med. 11 419-25

Leonard D W and Meek K M 1997 Refractive indices of the collagen fibrils and extrafibriller material of the corneal stroma Biophys. J. 72 1382-7

Liu Z and Pflugfelder S C 2000 The effects of long-term contact lens wear on corneal thickness, curvature, and surface regularity Ophthalmology 107 105-11

Maurice D N 1957 The structure and transparency of the cornea J. Physiol. 136 263-86

Munnerlyn C R, Koons S J and Marshall J 1988 Photorefractive keratectomy: a technique for laser refractive surgery J. Cataract Refract. Surg. 14 46-52

Neumann E G 1988 Single-Mode Fiber (Berlin: Springer) pp 50-4

Radhakrishnan S, Rollins A M, Roth J E, Yazdanfar S, Westphal V, Bardenstein D S and Izatt J A 2001 Real-time optical coherence tomography of the anterior segment at $1310 \mathrm{~nm}$ Arch. Ophthalmol. $1191179-85$

Sorin W V and Gray D F 1992 Simultaneous thickness and group index measurement using optical low-coherence reflectometry IEEE Photonics. Technol. Lett. 4 105-7

Tearney G J, Brezinski M E, Southern J F, Bouma B E, Hee M R and Fujimoto J G 1995 Determination of the refractive index of highly scattering human tissue by optical coherence tomography Opt. Lett. 20 2258-60

Wexler A 2000 Constant humidity solutions Handbook of Chemistry and Physics ed L Lide (Boca Raton, FL: CRC Press) section 15 